\title{
Application of Variational Iteration Method for Dropping Damage Evaluation of the Suspension Spring Packaging System
}

\author{
Shuang Song, ${ }^{1}$ Ning-ning Duan, ${ }^{1}$ and An-jun Chen ${ }^{1,2}$ \\ ${ }^{1}$ Department of Packaging Engineering, Jiangnan University, Wuxi 214122, China \\ ${ }^{2}$ China National Light Industry Package Quality Supervising \& Testing Center, Wuxi 214122, China \\ Correspondence should be addressed to An-jun Chen; caj62@163.com
}

Received 8 April 2014; Revised 9 June 2014; Accepted 12 June 2014; Published 25 June 2014

Academic Editor: Patricia J. Y. Wong

Copyright (c) 2014 Shuang Song et al. This is an open access article distributed under the Creative Commons Attribution License, which permits unrestricted use, distribution, and reproduction in any medium, provided the original work is properly cited.

\begin{abstract}
The dropping damage evaluation for packaging system is essential for safe transportation and storage. A dynamic model of nonlinear cubic-quintic Duffing oscillator for the suspension spring packaging system was proposed. Then, a first-order approximate solution was obtained by applying He's variable iteration method. Based on the results, a damage evaluation equation was derived, which reveals the main controlling physical parameters for damage potential of drop to packaged products concretely. Finally, the dropping damage boundary curves and surfaces for the system were discussed. It was found that decreasing the suspension angle can improve the safe region of the system.
\end{abstract}

\section{Introduction}

Newton [1] proposed the concept of damage boundary for the first time in 1968, which established the foundation of present cushioning packaging dynamics. However, it can only be applied to linear packaging systems. For nonlinear packaging systems, there has been some undergoing work since then. A dropping damage evaluation for a tangent nonlinear system with a critical component was proposed by Wang et al. [2]. Wang et al. [3] proposed a three-dimensional shock spectrum for nonlinear packaging system with a critical component. They also suggested the damage boundary surfaces concept for damage evaluation of a tangent nonlinear packaging system with a critical component [4]. The fatigue damage of most packaged products is caused by dropping shock in the process of transportation. Therefore, Wang $[5,6]$ proposed the concept of dropping damage boundary curve with system parameter and the dimensionless dropping shock velocity as two basic evaluation quantities. These theories are all based on numerical analysis method. However, the influence of relevant parameters cannot be revealed to show their physical significance clearly. The variational iteration method (VIM) proposed by He et al. [7-9], which has been widely applied [10-19], can solve many kinds of nonlinear equations without small parameters limitation (first-order approximate analytical solutions can achieve high precision). Wang et al. [20-22] studied the dropping response of typical nonlinear packaging systems and obtained inner-resonance conditions.

The suspension spring system with eight springs as cushioning components performs geometric nonlinearity and is suitable for protecting high precision instrument with low fragility. Wu and Yang [23] studied the natural vibration characteristics of the system under the excitation of foundational displacement. They found that the shock absorption performance of the system with pendulum springs was better than the one with vertical springs. Assuming the system was excited by rectangular pulse, the three-dimensional shock spectrum and the damage boundary surface were obtained by Wang and Chen [24-26]. They achieved the conclusion that both increasing the pulse incentive amplitude and decreasing the suspension angle could expand the safe area, and the safe area would be expanded more obviously when the angle was less than $75^{\circ}$.

In this paper, by applying the VIM, we solve the nondimensional dynamic equation of the suspension spring system under the excitation of dropping shock to obtain a firstorder approximate solution and obtain the nondimensional maximum acceleration expression. Then, a damage evaluation equation presenting the relationship between physical 


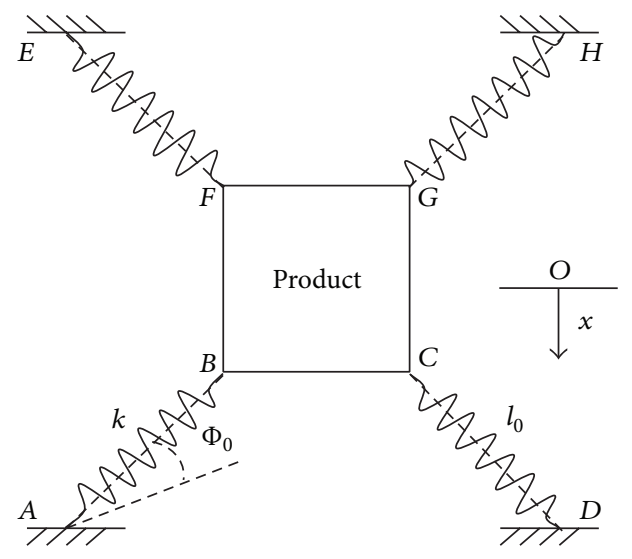

FIGURE 1: Dynamic model of the suspension spring system.

parameters and the damage boundary is suggested. Finally, the dropping damage boundary curves and surfaces of the system are discussed according to the damage evaluation equation.

\section{Modelling and Equations}

The dynamic model of the suspension spring packaging system is shown in Figure 1. A product is suspended in the middle of the container by 8 springs (four springs are on the upside, and the other four are on the downside).

Wang and Chen [24-26] proposed an approximate dynamic equation with a cubic oscillator of the suspension spring system. For acquiring a higher precision, we establish a more concrete dynamic model with nonlinear cubic-quintic Duffing oscillators of the system by using the Taylor series, which can be expressed as

$$
m \frac{\mathrm{d}^{2} x}{\mathrm{~d} t^{2}}+8 k\left(a_{0} x+\frac{b_{0}}{l_{0}^{2}} x^{3}+\frac{c_{0}}{l_{0}^{4}} x^{5}\right)=0
$$

where

$$
\begin{gathered}
a_{0}=\sin ^{2} \phi_{0}, \\
b_{0}=\frac{\left(1-6 \sin ^{2} \phi_{0}+5 \sin ^{4} \phi_{0}\right)}{2} \\
c_{0}=\frac{\left(63 \sin ^{6} \phi_{0}-105 \sin ^{4} \phi_{0}+45 \sin ^{2} \phi_{0}-3\right)}{8} .
\end{gathered}
$$

Based on dropping shock, the initial conditions can be written as

$$
\begin{gathered}
x(0)=0, \\
\frac{\mathrm{d} x(0)}{\mathrm{d} t}=\sqrt{2 g H} .
\end{gathered}
$$

Here are the coefficients: $H$ is the dropping height, $x$ denotes the product displacement, $m$ denotes the product mass, $g$ is the acceleration of gravity, $k$ denotes the coupling stiffness, $l_{0}$ denotes the original length of the springs, and $\phi_{0}$ denotes the suspension angle.

By introducing new nondimensional parameters, (1) can be equivalently written in the following dimensionless form:

$$
\frac{\mathrm{d}^{2} y}{\mathrm{~d} \tau^{2}}+a_{0} y+b_{0} y^{3}+c_{0} y^{5}=0,
$$

where

$$
\begin{gathered}
y=\frac{x}{l_{0}}, \\
\tau=\frac{t}{T}, \\
\omega=\sqrt{\frac{8 k}{m},} \\
T=\frac{1}{\omega} .
\end{gathered}
$$

The initial conditions can be written as

$$
\begin{gathered}
y(0)=0, \\
\frac{\mathrm{d} y(0)}{\mathrm{d} \tau}=V=\sqrt{\frac{2 g m H}{8 k l_{0}^{2}}},
\end{gathered}
$$

where $V$ denotes the dimensionless dropping velocity.

\section{Variational Iteration Method}

A nonlinear equation can be written as

$$
L y(t)+N y(t)=g(t),
$$

where $L$ is a linear operator, $N$ is a nonlinear operator, and $g$ is a continuous function.

The VIM is proposed by He et al. [7-9] for the first time, and the correction functional for the nonlinear equation can be established as follows:

$$
y_{n+1}=y_{n}(t)+\int_{0}^{t} \lambda(s)\left\{L y_{n}(s)+N \widetilde{y}_{n}(s)-g(s)\right\} d s,
$$

where $\lambda$ is the Laplace multiplier which can be obtained by the variational theory and $\widetilde{y}_{n}$ is the restriction on varying which is equal to 0 solving the Laplace multiplier.

Applying the VIM, construct the following correction functional of the system:

$$
\begin{aligned}
& y_{n+1}(\tau) \\
& =y_{n}(\tau) \\
& \quad+\int_{0}^{\tau} \lambda(s)\left(\frac{\mathrm{d}^{2} y_{n}(s)}{\mathrm{d} s^{2}}+a_{0} y_{n}(s)+b_{0} \tilde{y}_{n}^{3}(s)+c_{0} \tilde{y}_{n}^{5}(s)\right) \mathrm{d} s .
\end{aligned}
$$


According to the principle of stationary, (9) can be turned into the following form:

$$
\begin{aligned}
& \delta y_{n+1}(\tau) \\
& =\delta y_{n}(\tau) \\
& \quad+\delta \int_{0}^{\tau} \lambda(s)\left(\frac{\mathrm{d}^{2} y_{n}(s)}{\mathrm{d} s^{2}}+a_{0} y_{n}(s)+b_{0} \tilde{y}_{n}^{3}(s)+c_{0} \tilde{y}_{n}^{5}(s)\right) \mathrm{d} s \\
& =\delta y_{n}(\tau)+\delta \int_{0}^{\tau} \lambda(s) \ddot{y}_{n}(s) \mathrm{d} s+\delta \int_{0}^{\tau} \lambda(s) a_{0} y_{n}(s) \mathrm{d} s \\
& =\delta y_{n}(\tau)+\left.\lambda(s) \delta \dot{y}_{n}(s)\right|_{s=\tau}-\left.\dot{\lambda}(s) \delta y_{n}(s)\right|_{s=\tau} \\
& \quad+\int_{0}^{\tau}\left[\ddot{\lambda}(s)+a_{0} \lambda(s)\right] \cdot \delta y_{n}(s) \mathrm{d} s .
\end{aligned}
$$

The conditions of stationary can be written as

$$
\begin{gathered}
\ddot{\lambda}(s)+a_{0} \lambda(s)=0, \\
\left.\lambda(s)\right|_{s=\tau}=0, \\
\left.\dot{\lambda}(s)\right|_{s=\tau}=1 .
\end{gathered}
$$

The Laplace multiplier is obtained as

$$
\lambda(s)=\frac{1}{\sqrt{a_{0}}} \sin \left[\sqrt{a_{0}}(s-\tau)\right] .
$$

The following iteration formula can be constructed as

$$
\begin{aligned}
& y_{n+1}(\tau) \\
& =y_{n}(\tau) \\
& +\int_{0}^{\tau} \frac{1}{\sqrt{a_{0}}} \sin \left[\sqrt{a_{0}}(s-\tau)\right] \\
& \quad \cdot\left(\frac{\mathrm{d}^{2} y_{n}(s)}{\mathrm{d} s^{2}}+a_{0} y_{n}(s)+b_{0} y_{n}^{3}(s)+c_{0} y_{n}^{5}(s)\right) \mathrm{d} s .
\end{aligned}
$$

For the nondimensional dynamic equation (4) and the initial conditions equation (6), we can take the initial solution below:

$$
y_{0}(\tau)=A \sin \alpha \tau,
$$

where $\alpha$ is the frequency parameter and the amplitude $A=$ $V / \alpha$. The first-order iteration approximate solution can be obtained as

$$
\begin{aligned}
y_{1}(\tau)= & {\left[\frac{3 b_{0} A^{3}}{4\left(\alpha^{2}-a_{0}\right)}+\frac{5 c_{0} A^{5}}{8\left(\alpha^{2}-a_{0}\right)}\right] \sin \alpha \tau } \\
& +\left[-\frac{b_{0} A^{3}}{4\left(9 \alpha^{2}-a_{0}\right)}-\frac{5 c_{0} A^{5}}{16\left(9 \alpha^{2}-a_{0}\right)}\right] \sin 3 \alpha \tau \\
& +\left[\frac{c_{0} A^{5}}{16\left(25 \alpha^{2}-a_{0}\right)}\right] \sin 5 \alpha \tau
\end{aligned}
$$

$$
\begin{gathered}
+\left[\frac{A \alpha}{\sqrt{a_{0}}\left(\alpha^{2}-a_{0}\right)}\left(\alpha^{2}-a_{0}-\frac{3}{4} b_{0} A^{3}-\frac{5}{8} c_{0} A^{5}\right)\right. \\
+\frac{3 \alpha b_{0} A^{3}}{4 \sqrt{a_{0}}\left(9 \alpha^{2}-a_{0}\right)}+\frac{15 \alpha c_{0} A^{5}}{16 \sqrt{a_{0}}\left(9 \alpha^{2}-a_{0}\right)} \\
\left.\quad-\frac{5 \alpha c_{0} A^{5}}{16 \sqrt{a_{0}}\left(25 \alpha^{2}-a_{0}\right)}\right] \sin \sqrt{a_{0}} \tau .
\end{gathered}
$$

Let the coefficient of $\sin \sqrt{a_{0}} \tau$ be equal to zero so that there is no secular term appearing in the next iteration; namely,

$$
\begin{gathered}
\frac{A \alpha}{\sqrt{a_{0}}\left(\alpha^{2}-a_{0}\right)}\left(\alpha^{2}-a_{0}-\frac{3}{4} b_{0} A^{3}-\frac{5}{8} c_{0} A^{5}\right) \\
+\frac{3 \alpha b_{0} A^{3}}{4 \sqrt{a_{0}}\left(9 \alpha^{2}-a_{0}\right)}+\frac{15 \alpha c_{0} A^{5}}{16 \sqrt{a_{0}}\left(9 \alpha^{2}-a_{0}\right)} \\
-\frac{5 \alpha c_{0} A^{5}}{16 \sqrt{a_{0}}\left(25 \alpha^{2}-a_{0}\right)}=0 .
\end{gathered}
$$

As the dropping shock pulse is a half-sine pulse, the nondimensional dropping shock extended period can be obtained as

$$
\tau=\frac{\pi}{\alpha} .
$$

The first-order nondimensional displacement iteration approximate expression, namely, the first-order approximate solution, can be written as

$$
\begin{aligned}
y_{1}(\tau)= & {\left[\frac{3 b_{0} A^{3}}{4\left(\alpha^{2}-a_{0}\right)}+\frac{5 c_{0} A^{5}}{8\left(\alpha^{2}-a_{0}\right)}\right] \sin \alpha \tau } \\
& -\left[\frac{b_{0} A^{3}}{4\left(9 \alpha^{2}-a_{0}\right)}+\frac{5 c_{0} A^{5}}{16\left(9 \alpha^{2}-a_{0}\right)}\right] \sin 3 \alpha \tau \\
& +\left[\frac{c_{0} A^{5}}{16\left(25 \alpha^{2}-a_{0}\right)}\right] \sin 5 \alpha \tau .
\end{aligned}
$$

The first-order nondimensional acceleration iteration approximate expression can be written as

$$
\begin{aligned}
y_{1}^{\prime \prime}(\tau)= & -\alpha^{2}\left[\frac{3 b_{0} A^{3}}{4\left(\alpha^{2}-a_{0}\right)}+\frac{5 c_{0} A^{5}}{8\left(\alpha^{2}-a_{0}\right)}\right] \sin \alpha \tau \\
& +9 \alpha^{2}\left[\frac{b_{0} A^{3}}{4\left(9 \alpha^{2}-a_{0}\right)}+\frac{5 c_{0} A^{5}}{16\left(9 \alpha^{2}-a_{0}\right)}\right] \sin 3 \alpha \tau \\
& -25 \alpha^{2}\left[\frac{c_{0} A^{5}}{16\left(25 \alpha^{2}-a_{0}\right)}\right] \sin 5 \alpha \tau .
\end{aligned}
$$


Substitute $\alpha \tau=\pi / 2$ into (18) and (19); the nondimensional maximum displacement can be written as

$$
\begin{aligned}
y_{1}(\tau)_{m}=\mid\left[\frac{3 b_{0} A^{3}}{4\left(\alpha^{2}-a_{0}\right)}+\frac{5 c_{0} A^{5}}{8\left(\alpha^{2}-a_{0}\right)}\right] \\
+\left[\frac{b_{0} A^{3}}{4\left(9 \alpha^{2}-a_{0}\right)}+\frac{5 c_{0} A^{5}}{16\left(9 \alpha^{2}-a_{0}\right)}\right] \\
+\left[\frac{c_{0} A^{5}}{16\left(25 \alpha^{2}-a_{0}\right)}\right] \mid
\end{aligned}
$$

and the nondimensional maximum acceleration can be written as

$$
\begin{aligned}
y_{1}^{\prime \prime}(\tau)_{m}=\mid- & \alpha^{2}\left[\frac{3 b_{0} A^{3}}{4\left(\alpha^{2}-a_{0}\right)}+\frac{5 c_{0} A^{5}}{8\left(\alpha^{2}-a_{0}\right)}\right] \\
& -9 \alpha^{2}\left[\frac{b_{0} A^{3}}{4\left(9 \alpha^{2}-a_{0}\right)}+\frac{5 c_{0} A^{5}}{16\left(9 \alpha^{2}-a_{0}\right)}\right] \\
& -25 \alpha^{2}\left[\frac{c_{0} A^{5}}{16\left(25 \alpha^{2}-a_{0}\right)}\right] \mid
\end{aligned}
$$

For the following amounts, $m=290 \mathrm{~kg}, k=2 \times 10^{5} \mathrm{~N} / \mathrm{m}$, and $l_{0}=0.075 \mathrm{~m}$, we choose the dropping height $H=0.3 \mathrm{~m}$, and the suspension angle $\phi_{0}=60^{\circ}$. According to (16), we gain the frequency parameter $\alpha=0.8148$. By applying the VIM the nondimensional maximum acceleration and the dropping shock extended period are obtained as $y_{m 1}^{\prime \prime}=0.3298$ and $\tau_{1}=3.8555$. Applying the Runge-Kutta $(\mathrm{R}-\mathrm{K})$ method, results are $y_{m}^{\prime \prime}=0.3338$ and $\tau=3.8392$. Comparing with the R-K method, the relative errors by using the VIM are $E_{y_{m}^{\prime \prime}}=1.20 \%$ and $E_{\tau}=0.42 \%$, which can meet the requirement of the packaging design.

The impact energy under dropping shock is related not only to the maximum acceleration, but also to the whole waveform. Hence, it is necessary to prove that the overall precision of the waveform can meet the requirement. The nondimensional acceleration response curves can be obtained just as shown in Figure 2. It indicates that the curve by applying the VIM can meet very well with the one by the R-K method.

\section{Dropping Damage Evaluation}

Wang $[5,6]$ proposed the concept of dropping damaging boundary, which can provide the theoretical foundation for the dropping damage evaluation of the suspension spring system.

The dropping shock acceleration of the system can be written as follows:

$$
\frac{\mathrm{d}^{2} x}{\mathrm{~d} t^{2}}=\beta \frac{\mathrm{d}^{2} y}{\mathrm{~d} \tau^{2}},
$$

where the system parameter

$$
\beta=\frac{l_{0}}{T^{2}}=\frac{8 k l_{0}}{m}
$$

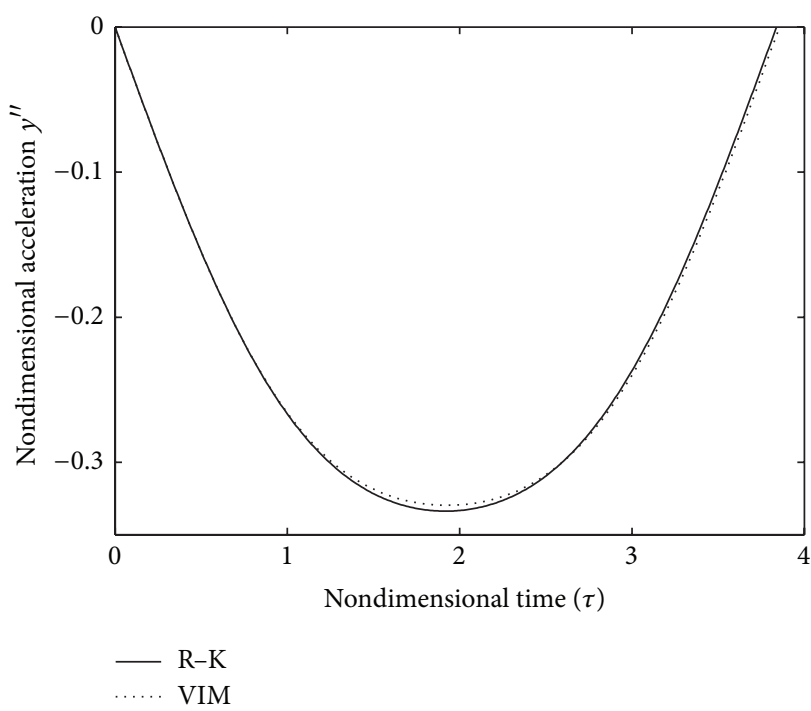

FIGURE 2: Comparison of the nondimensional acceleration $y^{\prime \prime}$-time $\tau$ response of the system by the VIM with the one by the R-K method when the suspension angle $\phi_{0}=60^{\circ}$.

Set $A_{c}$ as the product fragility. The relational expression about the nondimensional maximum acceleration $y_{m}^{\prime \prime}$, the product fragility $A_{c}$, and the system parameter $\beta$ can be obtained just as follows:

$$
A_{c} g=\left(\frac{\mathrm{d}^{2} x}{\mathrm{~d} t^{2}}\right)_{m}=\beta\left(\frac{\mathrm{d}^{2} y}{\mathrm{~d} \tau^{2}}\right)_{m}
$$

By combining (21) and (24), the system parameter can be written as

$$
\begin{aligned}
& \beta= A_{c} g \\
& \times\left(\mid-\alpha^{2}\left[\frac{3 b_{0} A^{3}}{4\left(\alpha^{2}-a_{0}\right)}+\frac{5 c_{0} A^{5}}{8\left(\alpha^{2}-a_{0}\right)}\right]\right. \\
&-9 \alpha^{2}\left[\frac{b_{0} A^{3}}{4\left(9 \alpha^{2}-a_{0}\right)}+\frac{5 c_{0} A^{5}}{16\left(9 \alpha^{2}-a_{0}\right)}\right] \\
&\left.-25 \alpha^{2}\left[\frac{5 c_{0} A^{5}}{16\left(25 \alpha^{2}-a_{0}\right)}\right] \mid\right)^{-1} .
\end{aligned}
$$

Equation (25) includes more than one variable such as $\beta$, $A_{c}, A$ (related to $V$ and $\alpha$ ), and $\phi_{0}$. Therefore, according to (25), we can evaluate the dropping shock characteristics of the system.

Respectively, we choose the suspension angle $\phi_{0}$, equal to $60^{\circ}, 65^{\circ}, 70^{\circ}$, and $75^{\circ}$, and select the product fragility $A_{c}$, equal to 10 and 15 . The system parameter $\beta$ and the dimensionless dropping shock velocity $V$ are selected as two basic evaluation quantities $[5,6]$. According to (25), dropping damage boundary curves of the system can be obtained just as Figure 3 indicated. The safe area is under the dropping damage boundary curve, and the product is safe when the coordinate point $(\beta, V)$ enters the area. 


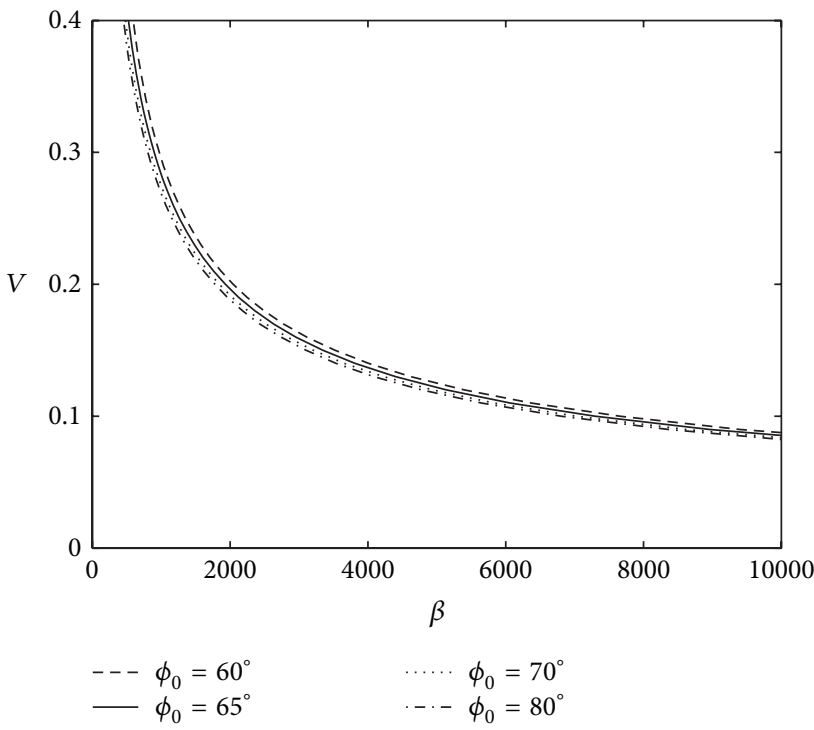

(a)

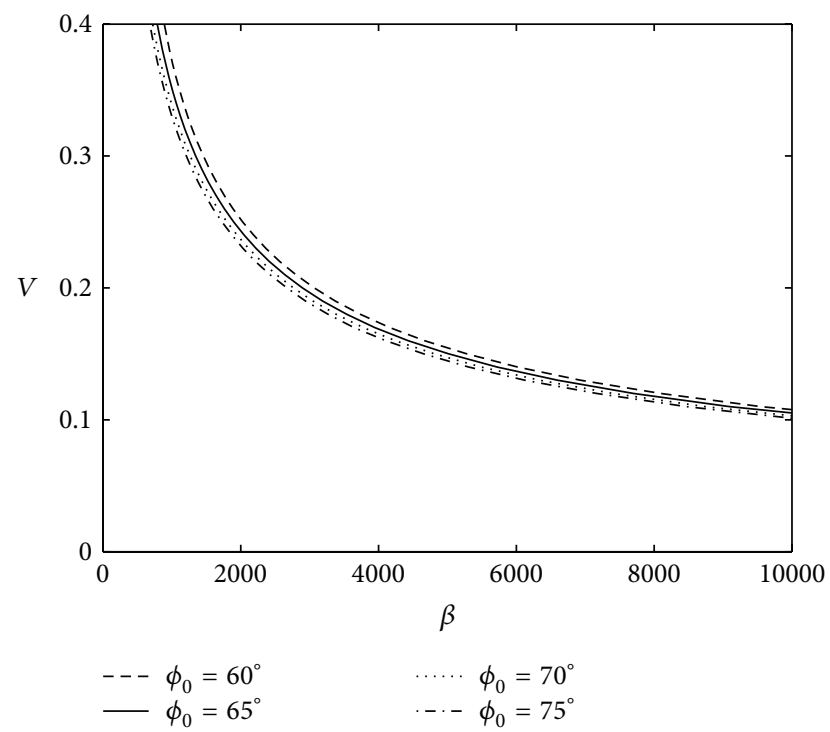

(b)

FIGURE 3: Dropping damage boundary curves of the system when the suspension angle $\phi_{0}=60^{\circ}, 65^{\circ}, 70^{\circ}$, and $75^{\circ}$ and the product fragility (a) $A_{c}=10$ and (b) $A_{c}=15 . V$ : the dimensionless dropping shock velocity; $\beta$ : the system parameter.

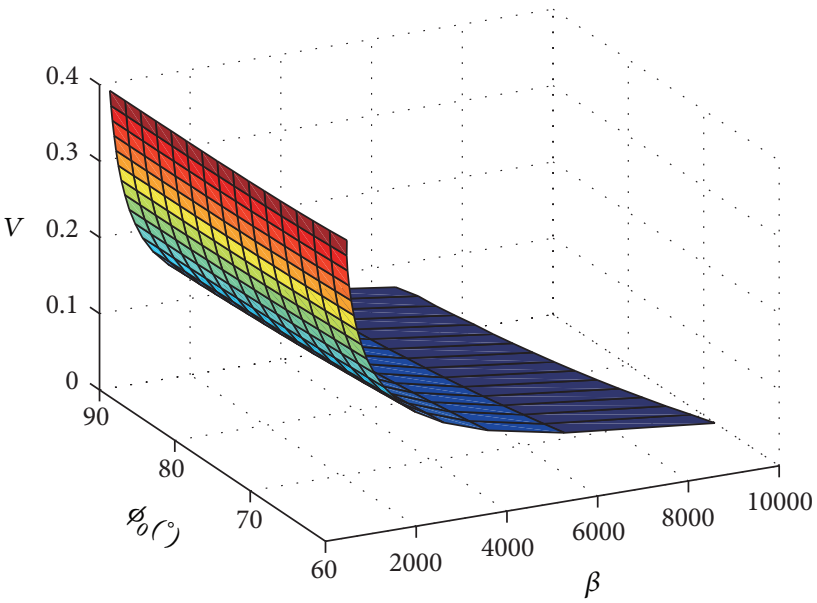

(a)

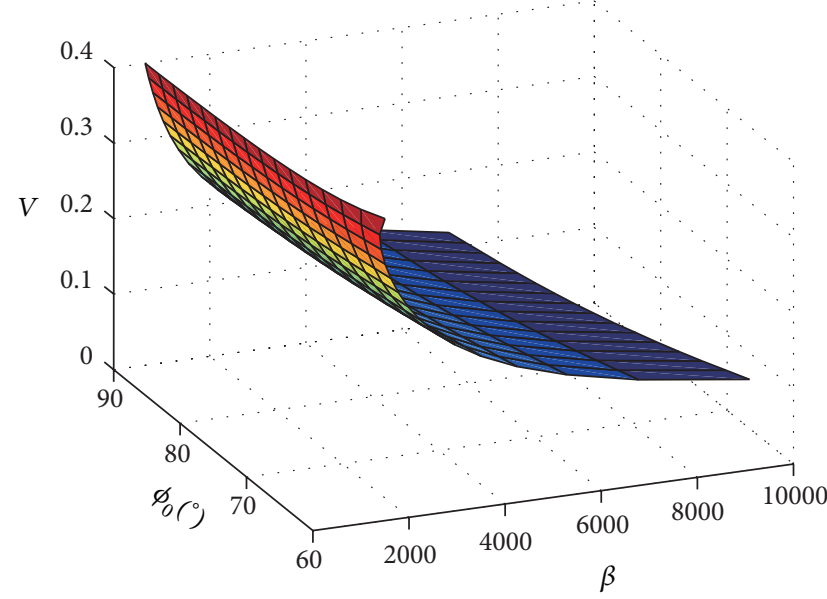

(c)

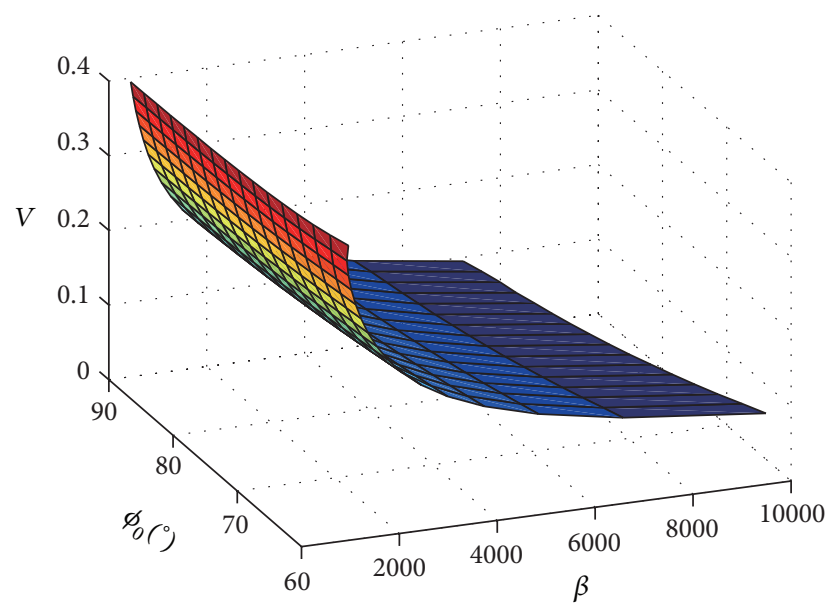

(b)

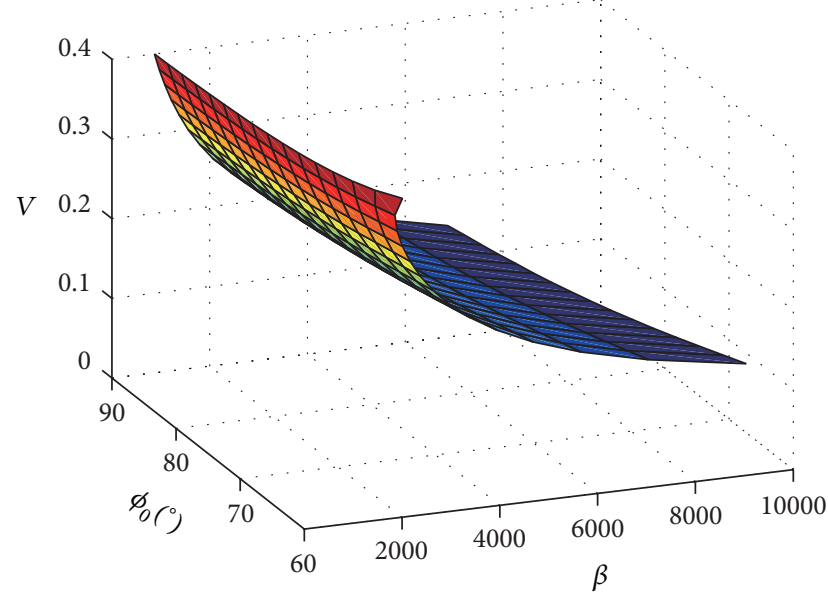

(d)

FIGURE 4: Dropping damage boundary surfaces of the system when the product fragility (a) $A_{c}=5$, (b) $A_{c}=10$, (c) $A_{c}=15$, and (d) $A_{c}=20$. $V$ : the dimensionless dropping shock velocity; $\beta$ : the system parameter; $\phi_{0}$ : the suspension angle. 
By selecting the suspension angle as the third evaluation quantity, set product fragility $A_{c}=5,10,15$, and 20, respectively, and then dropping damage boundary surfaces can be obtained by applying the VIM, just like Figure 4 indicated. The safe area is under the dropping damage boundary surfaces, and the product is safe when the coordinate point $\left(\beta, \phi_{0}, V\right)$ enters the area.

According to Figures 3 and 4, it is shown that decreasing the suspension angle $\phi_{0}$, the security performance of the system will improve, and increasing the product fragility $A_{c}$, the damage boundary curves and surface will move up obviously. So the characteristics of suspension geometry nonlinear $\left(\phi_{0}<90^{\circ}\right)$ to protect products are superior to the linear system $\left(\phi_{0}=90^{\circ}\right)$.

\section{Conclusion}

A dynamic model with nonlinear cubic-quintic Duffing oscillators is proposed for thesuspension spring packaging system, and the first-order approximate solution of the equation is obtained by He's variable iteration method. Based on the results, the damage evaluation equation for the packaging system is derived, revealing the main controlling parameters of the damage potential of dropping shock to packaged products. Finally, the damage boundary curves and surfaces for the system are discussed. It is found that decreasing the suspension angle can help to protect the packaged product.

\section{Conflict of Interests}

The authors declare that there is no conflict of interests regarding the publication of this paper.

\section{References}

[1] R. E. Newton, Fragility Assessment Theory and Practice, Monterey Research Laboratory, Monterey, Calif, USA, 1968.

[2] J. Wang, J. Jiang, L. Lu, and Z. Wang, "Dropping damage evaluation for a tangent nonlinear system with a critical component," Computers and Mathematics with Applications, vol. 61, no. 8, pp. 1979-1982, 2011.

[3] J. Wang, Z.-W. Wang, L.-X. Lu, Y. Zhu, and Y.-G. Wang, "Three-dimensional shock spectrum of critical component for nonlinear packaging system," Shock and Vibration, vol. 18, no. 3, pp. 437-445, 2011.

[4] J. Wang, F. Duan, J. Jiang, L. Lu, and Z. Wang, "Dropping damage evaluation for a hyperbolic tangent cushioning system with a critical component," Journal of Vibration and Control, vol. 18, no. 10, pp. 1417-1421, 2012.

[5] Z. Wang, "Dropping damage boundary curves for cubic and tangent package cushioning systems," Packaging Technology and Science, vol. 15, no. 5, pp. 263-266, 2002.

[6] Z. Wang, "On evaluation of product dropping damage," Packaging Technology and Science, vol. 15, no. 3, pp. 115-120, 2002.

[7] J. H. He and X. H. Wu, "Variational iteration method: new development and applications," Computer \& Mathematics with Applications, vol. 54, no. 7-8, pp. 881-894, 2007.

[8] J. He, "Variational iteration method-some recent results and new interpretations," Journal of Computational and Applied Mathematics, vol. 207, no. 1, pp. 3-17, 2007.
[9] J. H. He, G. C. Wu, and F. Austin, "The variational iteration method which should be followed," Nonlinear Science Letters A, vol. 1, no. 1, pp. 1-30, 2010.

[10] E. Yusufoglu, "The variational iteration method for studying the Klein-Gordon equation," Applied Mathematics Letters, vol. 21, no. 7, pp. 669-674, 2008.

[11] Z. Odibat and S. Momani, "The variational iteration method: an efficient scheme for handling fractional partial differential equations in fluid mechanics," Computers \& Mathematics with Applications, vol. 58, no. 11-12, pp. 2199-2208, 2009.

[12] D. D. Ganji and A. Sadighi, "Application of homotopyperturbation and variational iteration methods to nonlinear heat transfer and porous media equations," Journal of Computational and Applied Mathematics, vol. 207, no. 1, pp. 24-34, 2007.

[13] M. Rafei, H. Daniali, and D. D. Ganji, "Variational interation method for solving the epidemic model and the prey and predator problem," Applied Mathematics and Computation, vol. 186, no. 2, pp. 1701-1709, 2007.

[14] H. Shahmohamadi and M. M. Rashidi, "A novel solution for the glauert-jet problem by variational iteration method-padé approximant," Mathematical Problems in Engineering, vol. 2010, Article ID 501476, 7 pages, 2010.

[15] I. Kucuk, "Active optimal control of the KdV equation using the variational iteration method," Mathematical Problems in Engineering, vol. 2010, Article ID 929103, 10 pages, 2010.

[16] Y. Khan, H. Vázquez-Leal, and L. Hernandez-Martínez, "Removal of noise oscillation term appearing in the nonlinear equation solution," Journal of Applied Mathematics, vol. 2012, Article ID 387365, 9 pages, 2012.

[17] Y. Khan, H. Vázquez-Leal, and N. Faraz, "An efficient new iterative method for oscillator differential equation," Scientia Iranica, vol. 19, no. 6, pp. 1473-1477, 2012.

[18] Y. Khan, M. Madani, A. Yildirim, M. A. Abdou, and N. Faraz, "A new approach to Van der Pol's oscillator problem," Zeitschrift fur Naturforschung A: Journal of Physical Sciences, vol. 66, no. 10-11, pp. 620-624, 2011.

[19] Y. Khan, N. Faraz, A. Yildirim, and Q. B. Wu, "Fractional variational iteration method for fractional initial-boundary value problems arising in the application of nonlinear science," Computers \& Mathematics with Applications, vol. 62, no. 5, pp. 2273-2278, 2011.

[20] J. Wang, Y. Khan, R. Yang, L. Lu, Z. Wang, and N. Faraz, "A mathematical modelling of inner-resonance of tangent nonlinear cushioning packaging system with critical components," Mathematical and Computer Modelling, vol. 54, no. 11-12, pp. 2573-2576, 2011.

[21] J. Wang, Y. Khan, L. X. Lu, and Z. W. Wang, "Inner resonance of a coupled hyperbolic tangent nonlinear oscillator arising in a packaging system," Applied Mathematics and Computation, vol. 218, no. 15, pp. 7876-7879, 2012.

[22] J. Wang, Z. W. Wang, F. Duan, L. Lu, D. Gao, and A. Chen, "Dropping shock response of corrugated paperboard cushioning packaging system," Journal of Vibration and Control, vol. 19, no. 3, pp. 336-340, 2013.

[23] X. Wu and L. J. Yang, "Natural vibration characteristics of suspension spring geometric nonlinear damping system," Journal of Vibration and Shock, vol. 27, no. 11, pp. 71-72, 2008.

[24] L. Wang and A. J. Chen, "Shock characteristics of a suspension spring system under action of a rectangular pulse," Journal of Vibration and Shock, vol. 31, no. 11, pp. 142-144, 2012. 
[25] L. Wang and A. Chen, "The damage boundary curve of the suspension packaging system under rectangular pulse," Applied Mechanics and Materials, vol. 105-107, pp. 70-73, 2012.

[26] L. Wang and A. J. Chen, "The shock response spectra of the suspension packaging system under rectangular pulse," Journal of Applied Packaging Research, vol. 5, no. 4, pp. 237-246, 2011. 


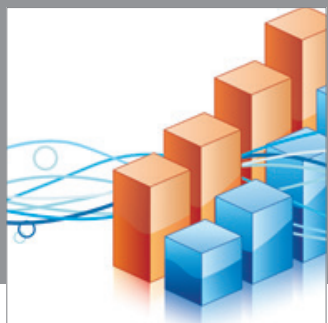

Advances in

Operations Research

mansans

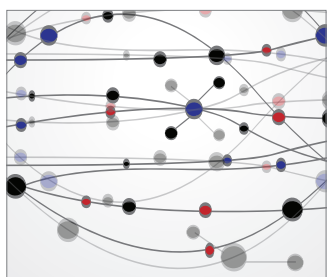

The Scientific World Journal
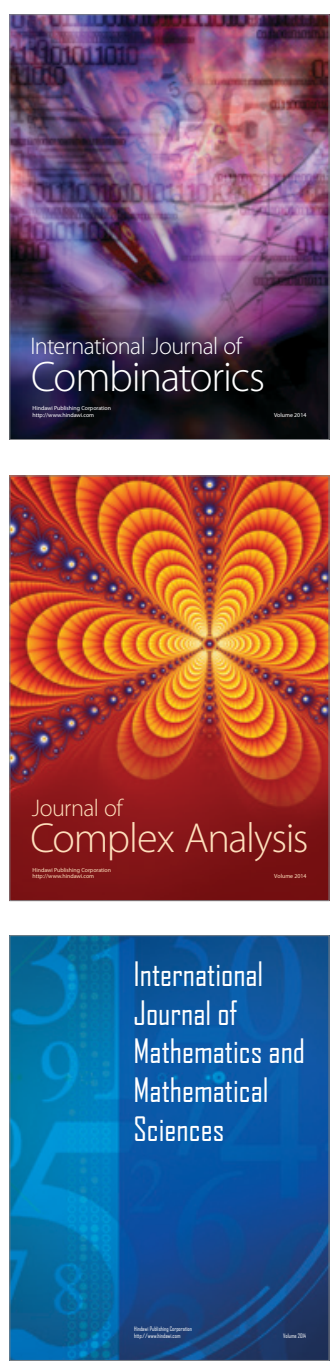
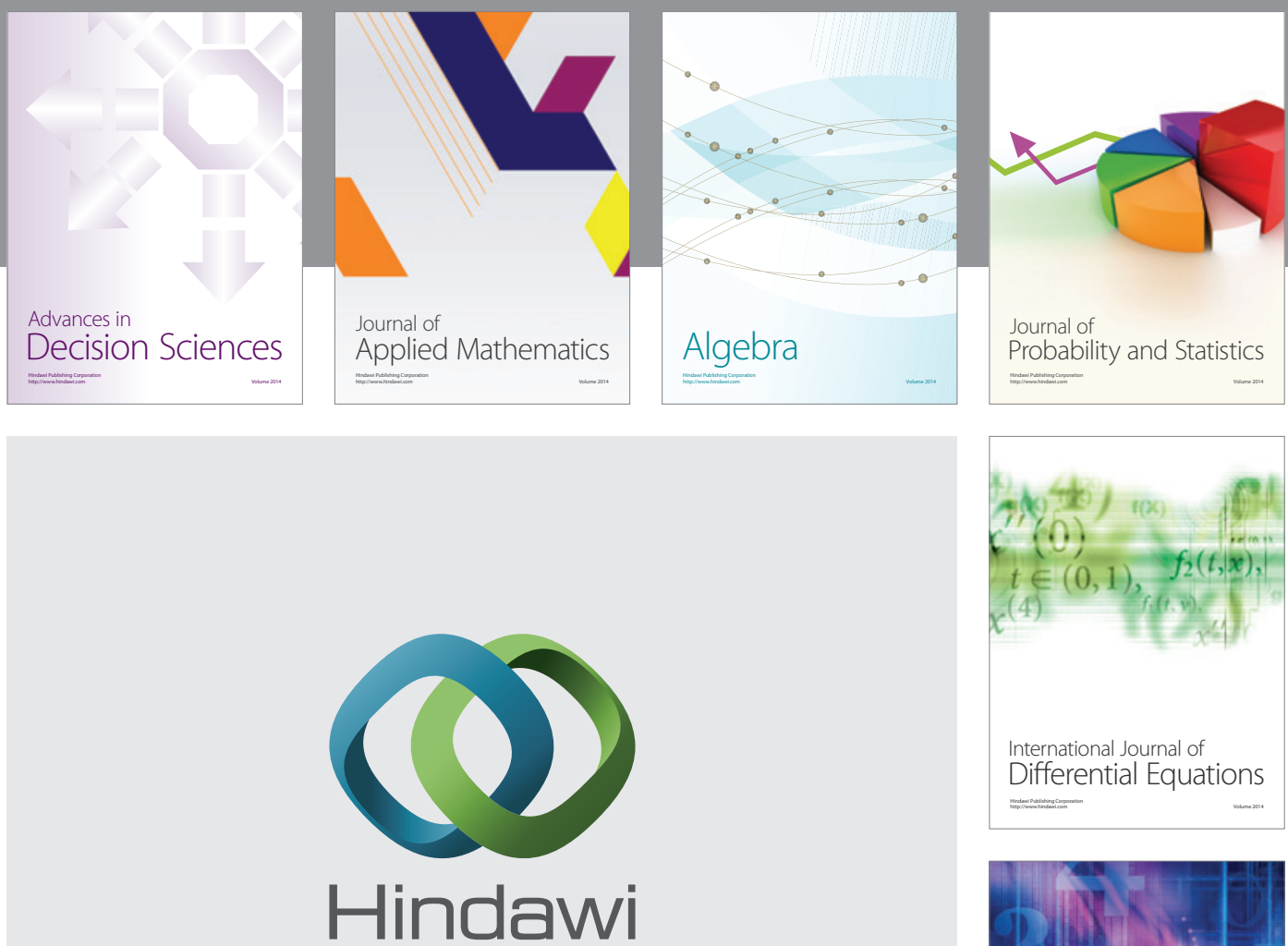

Submit your manuscripts at http://www.hindawi.com
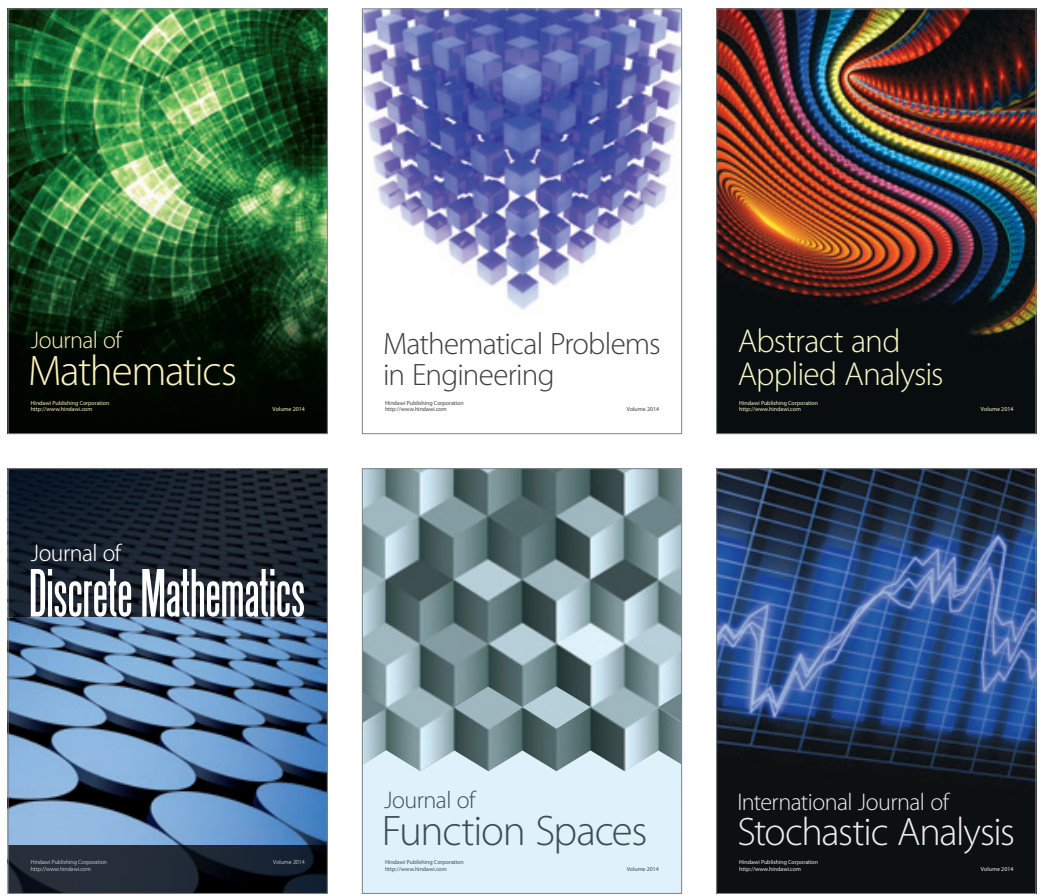

Journal of

Function Spaces

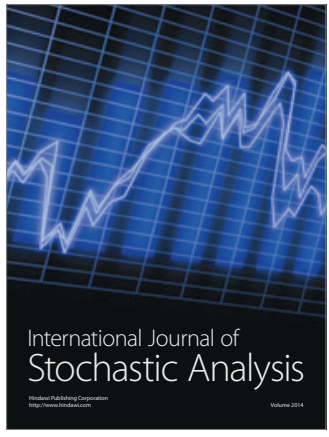

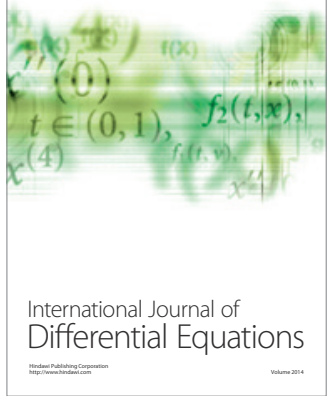
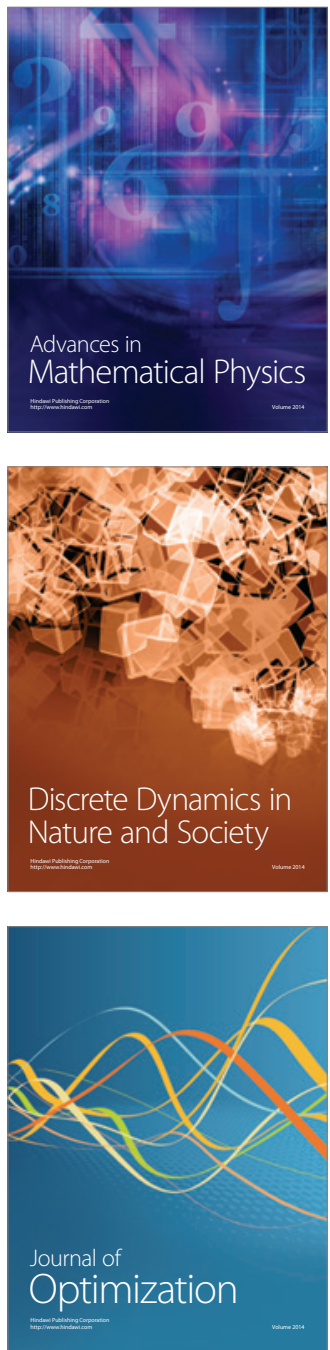